\title{
Robustness in projected entangled pair states
}

\author{
J. Ignacio Cirac, ${ }^{1}$ Spyridon Michalakis, ${ }^{2}$ David Pérez-García, ${ }^{3}$ and Norbert Schuch $^{4}$ \\ ${ }^{1}$ Max-Planck-Institut für Quantenoptik, Hans-Kopfermann-Str. 1, D-85748 Garching, Germany \\ ${ }^{2}$ Institute for Quantum Information and Matter, Caltech, Pasadena, California 91125, USA \\ ${ }^{3}$ Dpto. Análisis Matemático and IMI, Universidad Complutense de Madrid, 28040 Madrid, Spain \\ ${ }^{4}$ Institute for Quantum Information, RWTH Aachen University, D-52056 Aachen, Germany
}

(Received 16 July 2013; published 6 September 2013)

\begin{abstract}
We analyze a criterion which guarantees that the ground states of certain many-body systems are stable under perturbations. Specifically, we consider PEPS, which are believed to provide an efficient description, based on local tensors, for the low energy physics arising from local interactions. In order to assess stability in the framework of PEPS, one thus needs to understand how physically allowed perturbations of the local tensor affect the properties of the global state. In this paper, we show that a restricted version of the local topological quantum order (LTQO) condition [Michalakis and Pytel, Commun. Math. Phys. 322, 277 (2013)] provides a checkable criterion which allows us to assess the stability of local properties of PEPS under physical perturbations. We moreover show that LTQO itself is stable under perturbations which preserve the spectral gap, leading to nontrivial examples of PEPS which possess LTQO and are thus stable under arbitrary perturbations.
\end{abstract}

DOI: $10.1103 /$ PhysRevB.88.115108

PACS number(s): 71.10.-w, 03.65.Vf, 05.30.-d, 75.10.-b

\section{INTRODUCTION}

In studying model Hamiltonians for condensed matter systems, it is essential to understand the conditions which guarantee that properties behave nicely under small perturbations, as this allows us to use the model to predict the behavior of actual physical systems. In the context of zerotemperature physics, this amounts to understanding the conditions under which certain physical properties of the ground state change smoothly under perturbations to the Hamiltonian. While this question is very hard to answer in general, a proof of stability under arbitrary perturbations has recently been given for frustration-free Hamiltonians, ${ }^{1}$ based on two conditions (LTQO and local gap), following up on earlier work on commuting Hamiltonians., ${ }^{2,3}$ However, the LTQO condition, and especially the local gap condition, are very hard to check in practice, and so far, no examples beyond commuting Hamiltonians fulfilling these properties have been devised.

Projected entangled pair states (PEPS) provide a local description of quantum many-body states based on their entanglement structure, and thus in a natural way embody the physics of gapped local Hamiltonians. PEPS can be used as a framework to understand the physics of many-body systems based on the state (similar as, e.g., the Laughlin wave function), in particular since to any PEPS, a local parent Hamiltonian can be associated. In the case of translational invariant systems, the state is described by a single local tensor, and understanding any property of the system can be mapped to studying a corresponding property of this tensor. In this way, PEPS have been very successful in understanding otherwise intractable questions, such as the characterization of topological order from local symmetries, ${ }^{4}$ the way in which global symmetries emerge locally, ${ }^{5}$ or the characterization of quantum phases without and with symmetries in one dimension ${ }^{6-8}$ and beyond, ${ }^{9}$ just to name a few. To assess how general these results are, it is therefore important to identify the conditions under which PEPS are robust to perturbations. Given the state-centered perspective of the PEPS framework, we are particularly interested in those "natural" perturbations to the state which both correspond to a perturbation of the local tensor, and at the same time can be understood as arising from a perturbation of the parent Hamiltonian. Unfortunately, the powerful tools available to assess these questions in one dimension cannot be applied to two-dimensional systems, leaving the stability of PEPS in 2D and beyond an open problem.

In this paper, we study the robustness of PEPS under natural perturbations and show that the LTQO condition, when restricted to specific observables or regions, allows us to prove stability of physical properties for those observables or regions. In the context of PEPS, this restricted version of LTQO has several advantages: On the one hand, it allows us to check the stability of local observables, their derivatives, and correlation functions under natural perturbations. In particular, LTQO for an observable discards first and second order phase transitions, whereas LTQO for a region discards any finite order one. On the other hand, as it relies only on the properties of specific operators or regions, it can be verified numerically by reducing it to an eigenvalue problem. Finally, in the PEPS framework with its state-centered perspective, there is no need to additionally check spectral properties of the Hamiltonian, thereby avoiding this particularly difficult task. While the motivation for this work stems from the framework of PEPS, the stability result as such is independent of PEPS and can be used to assess stability of general quantum states against a class of physically motivated perturbations. Along the way, we prove that LTQO implies fast decay of correlations in the system and that LTQO is itself a stable property.

This paper is structured as follows: In Sec. II, we introduce the PEPS formalism and discuss which types of perturbations are natural in the context of PEPS and parent Hamiltonians. In Sec. III, we introduce the restricted LTQO condition and prove that systems which satisfy LTQO w.r.t. certain observables or regions exhibit robustness against perturbations. In Sec. IV, we discuss how the restricted LTQO condition can be verified for PEPS. We close in Sec. V by showing that LTQO 
itself is stable under perturbations, which in turn allows us to construct the first examples verifying LTQO without commuting Hamiltonians. In the appendix, we give a proof that injective matrix product states (MPS) satisfy LTQO, implying that they are stable against general perturbations.

\section{PEPS}

In this section, we introduce the formalism of PEPS and their associated parent Hamiltonians, and define the natural perturbations within this framework.

\section{A. Definition}

We start by recalling the definition and basic properties of PEPS. For the sake of simplicity of the exposition we will concentrate on translationally invariant PEPS $|\Psi\rangle$ on a square lattice, but the results also hold for nontranslationally invariant PEPS and any lattice geometry. Each PEPS is characterized by a tensor $A \equiv A_{\alpha, \beta, \gamma, \delta}^{s}$ (with a physical index $s=1, \ldots, d$ representing the spin states on a single site, and auxiliary indices $\alpha, \beta, \gamma, \delta=1, \ldots, D)$, such that $\left\langle s_{1}, \ldots, s_{N} \mid \Psi\right\rangle$ is determined by associating the tensor $A^{s_{n}}$ to each spin $n$, and contracting the auxiliary indices connected by the lattice, as shown in Fig. 1. The number of auxiliary indices depends on the lattice geometry. We fix it to four since we are focusing on the square lattice. For periodic boundary conditions, we also contract the indices on the right boundary with those on the left and the ones pointing up with those down. For open boundary conditions we can, for instance, set the auxiliary indices at the boundary to a fixed value.

\section{B. Parent Hamiltonian}

PEPS are ground states of local frustration free Hamiltonians, which are called parent Hamiltonians. Given a PEPS $|\Psi\rangle$, a parent Hamiltonian has the form:

$$
H=\sum_{k} h_{k},
$$

$$
A_{\alpha, \beta, \gamma, \delta}^{n}=\gamma-\underbrace{\alpha}_{\beta} \delta
$$

$X_{\alpha_{1}, \alpha_{2}, \beta_{1}, \beta_{2}, \gamma_{1}, \gamma_{2}, \delta_{1}, \delta_{2}}^{n_{1}, n_{2}, n_{n}, n_{4}}=$

$$
\gamma_{1}
$$

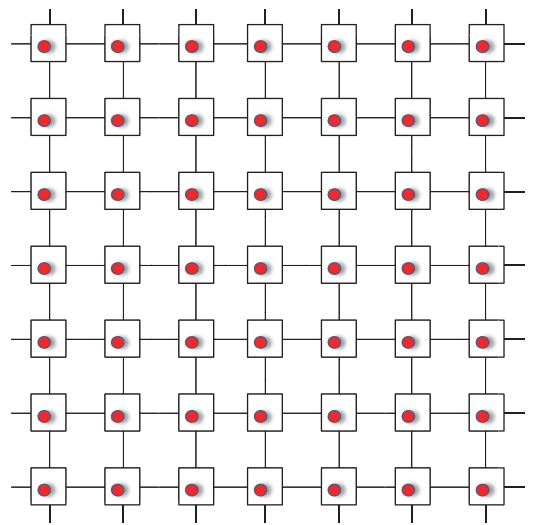

FIG. 1. (Color online) Top left: The tensor $A$ has a physical index $s$ (represented by a red point) and four auxiliary indices, $\alpha, \beta, \gamma, \delta$. The PEPS is built by contracting all the physical indices along the lattice, as it is represented by the drawing on the right. At the bottom left, we have the definition of the tensor $X$ as a contraction of four tensors $A$ along the auxiliary indices used to define the parent Hamiltonian. where each $h_{k} \geqslant 0$ acts on a finite region $k$ of the lattice. In order to understand the form of $h_{k}$, we introduce a subspace $\mathcal{K}_{k}$ corresponding to the spins in that region. This is the space spanned by all states $\left|\phi_{X}\right\rangle$ which are generated by contracting the tensors $A$ on region $k$, while assigning all possible values to the external auxiliary indices. For instance, if region $k$ is a plaquette composed of $2 \times 2$ spins (see Fig. 1), then:

$$
\mathcal{K}_{k}=\operatorname{span}\left\{\left|\phi_{\alpha_{1} \alpha_{2} \beta_{1} \beta_{2} \gamma_{1} \gamma_{2} \delta_{1} \delta_{2}}\right\rangle, 1 \leqslant \alpha_{i}, \beta_{i}, \gamma_{i}, \delta_{i} \leqslant D\right\},
$$

where

$$
\left|\phi_{\alpha_{1} \alpha_{2} \beta_{1} \beta_{2} \gamma_{1} \gamma_{2} \delta_{1} \delta_{2}}\right\rangle=\sum_{n_{1}, n_{2}, n_{3}, n_{4}} X_{\alpha_{1}, \alpha_{2}, \beta_{1}, \beta_{2}, \gamma_{1}, \gamma_{2}, \delta_{1}, \delta_{2}}^{n_{1}, n_{2}, n_{3}, n_{4}}\left|n_{1} n_{2} n_{3} n_{4}\right\rangle .
$$

For $H$ to be a parent Hamiltonian, $\mathcal{K}_{k}$ must coincide with the kernel of $h_{k}$. Furthermore, there must be a way of "growing" the regions $k$ step by step, such that: (i) at each step of joining the spins in neighboring regions, the so-called intersection property is fulfilled; ${ }^{10}$ (ii) the procedure terminates with a single region containing all spins. The intersection property simply states that if we join two regions $k_{1}$ and $k_{2}$ that intersect in some region $k$ (see Fig. 2), then:

$$
\left[\mathcal{K}_{k_{1}} \otimes \mathcal{H}_{k_{2} \backslash k}\right] \cap\left[\mathcal{H}_{k_{1} \backslash k} \otimes \mathcal{K}_{k_{2}}\right]=\mathcal{K}_{k_{1} k_{2}}
$$

Figure 2 gives an example of such a construction, where regions are composed of all possible square plaquettes, and regions $k_{1}$ and $k_{2}$ are overlapping plaquettes with two spins in common.

The fact that $\mathcal{K}_{k}$ coincides with the kernel of $h_{k}$ ensures that we have a frustration free Hamiltonian, i.e., $h_{k}|\Psi\rangle=0$. The other properties related to the growth ensure that for any region obtained in the intermediate steps, the ground-state subspace of the part of $H$ acting on that region is spanned by the tensors making up the PEPS if we contract them with arbitrary tensors

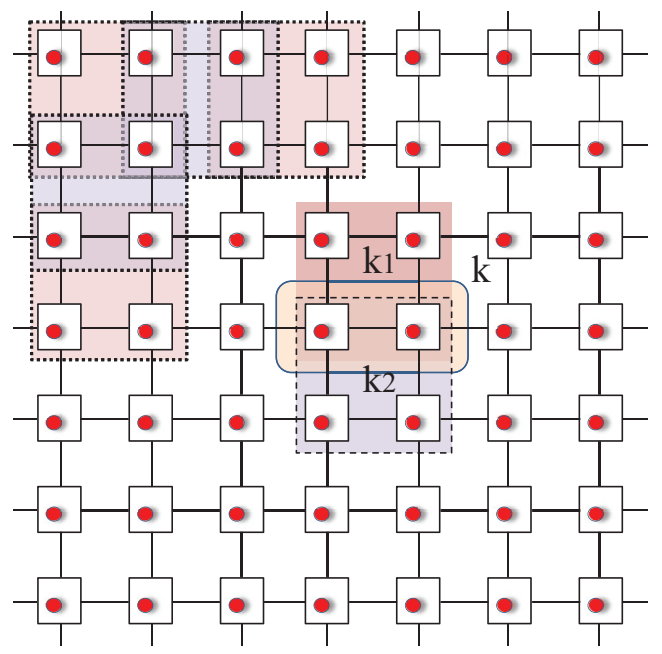

FIG. 2. (Color online) Construction of parent Hamiltonians for PEPS: The operator $h_{k_{1}}$ acts on a region $k_{1}$ (red region), and its kernel is spanned by all states that can be obtained by contracting the tensors $A$ on that region with arbitrary boundary conditions on the outgoing auxiliary indices. We can grow the regions by joining two regions, $k_{1}$ and $k_{2}$ (inside the dashed line) that intersect in $k$ (solid line). We can also block 4 neighboring spin as indicated by the red regions to form larger spins. 
at the boundary of that region; furthermore, the reduced state of $|\Psi\rangle$ on that region will be supported in that subspace. ${ }^{11}$

Note that there may be many parent Hamiltonians for a PEPS. On the one hand, any Hamiltonian built up from $\tilde{h}_{k} \geqslant 0$ which are fully supported in $\mathcal{K}_{k}^{\perp}$ will be a parent Hamiltonian, and on the other hand, we can choose different regions $k$ (for instance, containing a larger number of spins) to build $H$. The intersection property ensures that no unwanted states appear in the ground-state subspace once we have generated the full Hamiltonian.

\section{Perturbations}

The main aim of this paper is the study of the stability of properties of physical systems under perturbations, using the framework of PEPS. In what follows, we introduce the class of natural perturbations we will study in the context of PEPS.

The most obvious way of perturbing a PEPS is by replacing each tensor in the following way:

$$
A \rightarrow A(\epsilon)=A+\epsilon C, \quad\|C\|=1,
$$

possibly after blocking regions of tensors. Yet, such a perturbation can lead to a discontinuity in the parent Hamiltonian, ${ }^{14,15}$ and is therefore unphysical. Therefore, we will restrict Eq. (1) to the following class of natural perturbations, which can be understood as arising from a continuous perturbation of the parent Hamiltonian. ${ }^{12}$

Definition 1. The natural perturbations of a PEPS $|\Psi\rangle$ are those obtained by applying operators $R(\epsilon)$, with $\lim _{\epsilon \rightarrow 0} R(\epsilon)=\mathbb{1}$, to fully covering, but nonoverlapping, regions of bounded size, i.e.,

$$
|\Psi(\epsilon)\rangle=R(\epsilon)^{\otimes N^{\prime}}|\Psi\rangle,
$$

where $N^{\prime}$ is the number of regions.

To motivate our choice, note that perturbations of the form (2) can be understood as arising from a smooth perturbation of the parent Hamiltonian. ${ }^{8,17}$ To see why, start from the frustration-free parent Hamiltonian $H=\sum_{k} h_{k}$ for $|\Psi\rangle$ and let

$$
h_{k}(\epsilon)=\left(\left(R(\epsilon)^{-1}\right)^{\otimes \kappa}\right)^{\dagger} h_{k}\left(\left(R(\epsilon)^{-1}\right)^{\otimes \kappa}\right),
$$

where the product $\otimes \kappa$ goes over the sites on which $h_{k}$ acts. Note that $R(\epsilon)$ is invertible for small enough $\epsilon$, and $\lim _{\epsilon \rightarrow 0} h_{k}(\epsilon)=h_{k}$. Then,

$$
H(\epsilon)=\sum_{k} h_{k}(\epsilon)
$$

satisfies $h_{k}(\epsilon) \geqslant 0$ and $h_{k}(\epsilon)|\Psi(\epsilon)\rangle=0$, i.e., $|\Psi(\epsilon)\rangle$ is a ground state of the frustration-free Hamiltonian $H(\epsilon)$. Indeed, $H(\epsilon)$ is a parent Hamiltonian for $|\Psi(\epsilon)\rangle$ : The $h_{k}(\epsilon)$ have kernels $\mathcal{K}_{k}(\epsilon)=R(\epsilon)^{\otimes \kappa} \mathcal{K}_{k}$, which, since $R(\epsilon)$ is invertible, satisfy the conditions required for parent Hamiltonians discussed in Sec. II B. Note that the construction for $H(\epsilon)$ does not rely on $|\Psi\rangle$ being a PEPS, but only on $H$ being frustration free, thus our notion of natural perturbations applies to all frustration-free systems.

\section{THE LTQO CONDITION}

In this section, we recall the LTQO condition of Ref. 1 and define its restriction to particular observables and regions, which will be the desired checkable property ensuring stability in the context of PEPS without any spectral assumption. Since the condition can be introduced and analyzed for general systems (out of the context of PEPS), we will do so for the sake of generality.

We consider a spin lattice $X$ in arbitrary spatial dimension and lattice geometry, with corresponding Hilbert space $\mathcal{H}_{X}$. We will consider connected regions of the lattice $B$ with smooth boundaries $\partial B$ (see Fig. 3), and denote by $|B|$ the number of lattice points in that region and by $\mathcal{H}_{B}$ the corresponding Hilbert space for the spins. We assume a short-range, translationally invariant and frustration-free Hamiltonian $H_{X}$ acting on the lattice. We are interested in the properties of the ground-state subspace $\mathcal{S}_{X} \in \mathcal{H}_{X}$ in the limit $|X| \rightarrow \infty$.

We can write $H_{X}=H_{B}+H_{X \backslash B}+H_{\partial B}$, where $H_{Y}$ includes the terms of $H_{X}$ acting on $Y$, and denote by $\mathcal{S}_{B} \subset \mathcal{H}_{B}$ the ground-state subspace of $H_{B}$. Note that since $H_{X}$ is frustration free, all states in $\mathcal{S}_{X}$ are spanned by vectors in $\mathcal{S}_{B} \otimes \mathcal{S}_{X \backslash B}$

The LTQO property is related to the sensitivity of local observables to changes in the ground state far away from the region on which the observable acts. To define it, we divide the lattice $X$ into regions $A \subset B \subset X$, with $A$ and $B$ connected and finite, and denote by $m$ the distance between $A$ and $\partial B$ (see Fig. 3).

Definition 2. We say that a region $A$ satisfies LTQO, if for all observables $O_{a}$ supported on $A \subset X$, all regions $B \subset X$ with $A \subset B$ and ground states $\left|\Psi_{x}\right\rangle \in \mathcal{S}_{X}$, and $\left|\Psi_{b}\right\rangle \in \mathcal{S}_{B}$, the following bound holds:

$$
\left|\frac{\left\langle\Psi_{x}\left|O_{a}\right| \Psi_{x}\right\rangle}{\left\langle\Psi_{x} \mid \Psi_{x}\right\rangle}-\frac{\left\langle\Psi_{b}\left|O_{a}\right| \Psi_{b}\right\rangle}{\left\langle\Psi_{b} \mid \Psi_{b}\right\rangle}\right| \leqslant\left\|O_{a}\right\| f_{A}(m),
$$

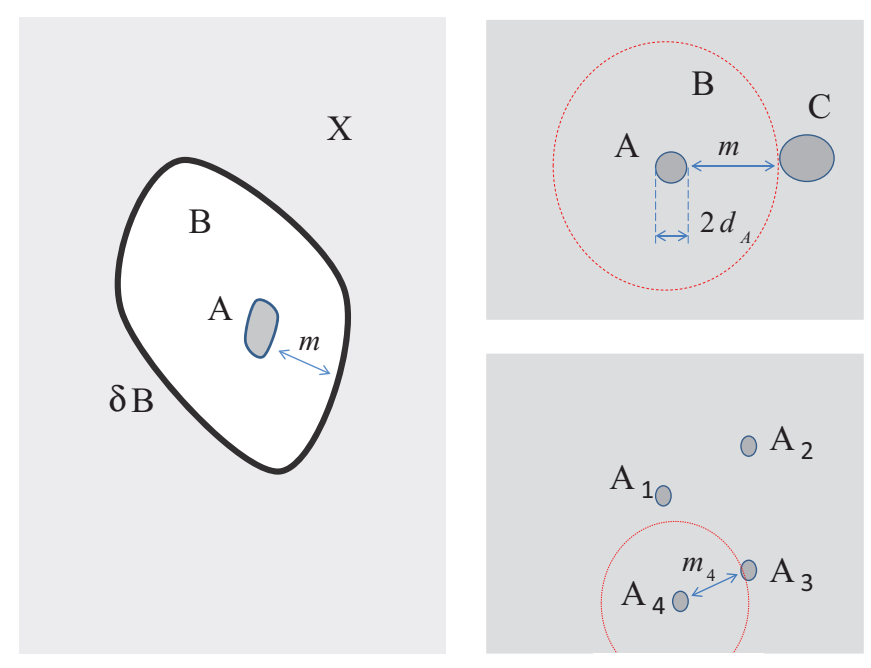

FIG. 3. (Color online) Left: Setup for the definition of LTQO (Definition 2). Right: Setup for the decay of two-body correlations, Proposition 4 (top), and many-body correlations, Proposition 5 (bottom). 
where $m$ is the distance between $A$ and $\partial B$ (cf. Fig. 3), and $f_{A}(m)$ decays superpolynomially in $m$, i.e.,

$$
\lim _{m \rightarrow \infty} p(m) f_{A}(m)=0,
$$

for all polynomials $p(m)$.

We say that a particular observable satisfies LTQO if it verifies (4).

We finally say that a system satisfies LTQO if all its regions $A$ satisfy it and the function $f$ in (4) is independent of $A$.

LTQO for a system was introduced in Ref. 1. Definition 2 adds its specialization for particular regions and observables, which will be crucial for this paper. Since we are assuming translational invariance, the exact position of region $A$ on the lattice does not play any role; only its shape matters. Moreover, since LTQO is inherited by subregions, one may restrict to regular shapes, like spheres or cubes. For the purposes of this work, we may think of region $A$ as a single site.

Proposition 3. The following three are equivalent definitions for LTQO, up to a possible prefactor in $f_{A}(m)$ :

(1) For all $\left|\Psi_{b}\right\rangle,\left|\Psi_{b}^{\prime}\right\rangle \in \mathcal{S}_{B}$,

$$
\left|\frac{\left\langle\Psi_{b}^{\prime}\left|O_{a}\right| \Psi_{b}^{\prime}\right\rangle}{\left\langle\Psi_{b}^{\prime} \mid \Psi_{b}^{\prime}\right\rangle}-\frac{\left\langle\Psi_{b}\left|O_{a}\right| \Psi_{b}\right\rangle}{\left\langle\Psi_{b} \mid \Psi_{b}\right\rangle}\right| \leqslant\left\|O_{a}\right\| f_{A}(m) .
$$

(2) For all density operators $\rho_{x}$ and $\rho_{b}$ supported in $\mathcal{S}_{X}$ and $\mathcal{S}_{B}$, respectively,

$$
\left|\operatorname{tr}\left(\rho_{x} O_{a}\right)-\operatorname{tr}\left(\rho_{b} O_{a}\right)\right| \leqslant\left\|O_{a}\right\| f_{A}(m) .
$$

(3) With $P_{B}$ the projector onto $\mathcal{S}_{B}$ and $c\left(O_{a}\right)=\frac{\operatorname{tr}\left(P_{B} O_{a}\right)}{\operatorname{tr}\left(P_{B}\right)}$,

$$
\left\|P_{B} O_{a} P_{B}-c\left(O_{a}\right) P_{B}\right\| \leqslant\left\|O_{a}\right\| f_{A}(m) .
$$

Moreover, the following slightly weaker condition is implied by LTQO:

(4) For all operators $Z$ acting on $X \backslash B$, and all $\left|\Psi_{x}\right\rangle \in \mathcal{S}_{X}$,

$$
\left|\frac{\left\langle\Psi_{x}\left|O_{a}\right| \Psi_{x}\right\rangle}{\left\langle\Psi_{x} \mid \Psi_{x}\right\rangle}-\frac{\left\langle\Psi_{x}^{\prime}\left|O_{a}\right| \Psi_{x}^{\prime}\right\rangle}{\left\langle\Psi_{x}^{\prime} \mid \Psi_{x}^{\prime}\right\rangle}\right| \leqslant\left\|O_{a}\right\| f_{A}(m),
$$

where we have defined $\left|\Psi_{x}^{\prime}\right\rangle=Z\left|\Psi_{x}\right\rangle$.

Proof. That (4) implies (5) is a simple use of the triangle inequality and accordingly changing $f$ by $2 f$. The reverse implication follows immediately if we write

$$
\frac{\operatorname{tr}_{X \backslash B}\left[\left|\Psi_{x}\right\rangle\left\langle\Psi_{x}\right|\right]}{\left\langle\Psi_{x} \mid \Psi_{x}\right\rangle}=\sum_{k} p_{k}\left|\Psi_{b}^{k}\right\rangle\left\langle\Psi_{b}^{k}\right|,
$$

where $\left|\Psi_{b}^{k}\right\rangle \in \mathcal{S}_{b}, p_{k} \geqslant 0$, and $\sum p_{k}=1$. That (6) implies (4) is obvious. The converse follows directly if we write the spectral decomposition of $\rho_{x}$ and $\rho_{b}$ and use the convexity of the absolute value. The equivalence between (6) and (7) can be seen following the steps of Corollary 3 in Ref. 1. Finally, that (6) implies (8) can be immediately seen by defining $\rho_{b}=$ $R_{b} / \operatorname{tr}\left(R_{b}\right)$ with $R_{b}=\operatorname{tr}_{X \backslash B}\left(\left|\Psi_{x}^{\prime}\right\rangle\left\langle\Psi_{x}^{\prime}\right|\right)$, so that

$$
\frac{\left\langle\Psi_{x}^{\prime}\left|O_{a}\right| \Psi_{x}^{\prime}\right\rangle}{\left\langle\Psi_{x}^{\prime} \mid \Psi_{x}^{\prime}\right\rangle}=\operatorname{tr}_{B}\left(\rho_{b} O_{a}\right)
$$

and noting that $\rho_{b}$ is supported in $\mathcal{S}_{B}$.

Note that if all vectors in $\mathcal{S}_{X}$ are fully supported on $\mathcal{S}_{B}$, then for all $\left|\Psi_{b}\right\rangle \in \mathcal{S}_{B}$ and $\left|\Psi_{x}\right\rangle \in \mathcal{S}_{X}$, there exists a vector $\left|\Psi_{y}\right\rangle \in$ $\mathcal{S}_{X \backslash B}$ such that $\left|\Psi_{b}\right\rangle=\left\langle\Psi_{y} \mid \Psi_{x}\right\rangle$ and thus (8) also implies (4). This occurs, for instance, if $H_{X}$ is the parent Hamiltonian of an injective (or more generally $G$-injective) PEPS. ${ }^{4,13}$
Some remarks are in order: (i) All conditions have to be fulfilled independently of the lattice size $|X|$, and therefore also in the thermodynamic limit. (ii) Equation (4) implies that in the thermodynamic limit, no two states in $\mathcal{S}_{X}$ can be distinguished locally by means of $O_{a}$. (iii) Equation (5) implies that $O_{a}$ cannot distinguish different states in $\mathcal{S}_{B}$, as long as the boundary of $B$ is far enough from the region where we measure. (iv) We will, in the following, generally assume that $B$ is spherical. Indeed, (4) cannot be modified to depend on $|B|$ (and thus the shape of $B$ ) in a nontrivial way: On the one hand, an exponential dependence on $|B|$ would override the scaling of $f(m)$ and invalidate the condition. On the other hand, a polynomial scaling $p(|B|)$ can be removed by choosing a spherical $B^{\prime} \subset B$ with identical $m$, and observing that in (6), $\left|\operatorname{tr}\left(\rho_{x} O_{a}\right)-\operatorname{tr}\left(\rho_{b} O_{a}\right)\right|=\left|\operatorname{tr}\left(\rho_{x} O_{a}\right)-\operatorname{tr}\left(\rho_{b^{\prime}} O_{a}\right)\right| \leqslant$ $\left\|O_{a}\right\| p\left(\left|B^{\prime}\right|\right) f_{A}(m)$, where $p\left(\left|B^{\prime}\right|\right)$ is polynomial in $m$ and can thus be absorbed in $f$.

\section{IMPLICATIONS OF THE LTQO CONDITION}

We will now analyze which restrictions the LTQO condition imposes on a system. We start by showing a superpolynomial decay of correlations and then use this to give the desired stability result.

\section{A. Correlation functions}

We show here that if an observable $O_{a}$ satisfies LTQO, then correlation functions must decay superpolynomially ${ }^{18}$ with the distance.

Proposition 4. If $O_{a}$ satisfies LTQO, then for any observable $O_{b}$ acting on $X \backslash B$ (cf. Fig. 3),

$$
\left|\left\langle O_{a} O_{b}\right\rangle-\left\langle O_{a}\right\rangle\left\langle O_{b}\right\rangle\right| \leqslant\left\|O_{a}\right\|\left\|O_{b}\right\| f_{A}(m),
$$

where the expectation value is taken in any normalized state $\left|\Psi_{x}\right\rangle \in \mathcal{S}_{X}$.

Proof. We can always write $O_{b}=P_{b}-Q_{b}$, where both $P_{b}, Q_{b} \geqslant 0$ and $\left\|O_{b}\right\|=\max \left\{\left\|P_{b}\right\|,\left\|Q_{b}\right\|\right\}$, so that we just have to prove (9) for $P_{b} \geqslant 0$. Defining $\left|\Psi_{x}^{\prime}\right\rangle=\sqrt{P_{b}}\left|\Psi_{x}\right\rangle$, we have

$$
\left\langle O_{a} P_{b}\right\rangle=\frac{\left\langle\Psi_{x}^{\prime}\left|O_{a}\right| \Psi_{x}^{\prime}\right\rangle}{\left\langle\Psi_{x}^{\prime} \mid \Psi_{x}^{\prime}\right\rangle}\left\langle\Psi_{x}\left|P_{b}\right| \Psi_{x}\right\rangle .
$$

Using condition (8) and the fact that the last factor is bounded by $\left\|P_{b}\right\|$, we obtain (9) (up to a factor of 2).

We can iterate Eq. (9) to prove that also many-site correlation functions decay fast. To this end, let us consider some regions $A_{1}, A_{2}, \ldots, A_{M}$, and denote by $m_{k}$ the shortest distance between $A_{k}$ and the rest of the regions (see Fig. 3 ). Then,

Proposition 5. For any set of observables $O_{a_{k}}$ verifying LTQO and acting on regions $\mathrm{A}_{k}$,

$$
\left|\left\langle\prod_{k=1}^{M} O_{a_{k}}\right\rangle-\prod_{k=1}^{M}\left\langle O_{a_{k}}\right\rangle\right| \leqslant \prod_{k=1}^{M}\left\|O_{a_{k}}\right\| \sum_{k=1}^{M} f_{A_{k}}\left(m_{k}\right) .
$$

Proof. We define

$$
r_{n}=\left\langle\prod_{k=1}^{n} O_{a_{k}}\right\rangle-\left\langle\prod_{k=1}^{n-1} O_{a_{k}}\right\rangle\left\langle O_{a_{n}}\right\rangle, \quad s_{n}=\prod_{k+1}^{M}\left\langle O_{a_{k}}\right\rangle,
$$


with $s_{M}=1$ and $r_{1}=0$. We have that

$$
\left|\left\langle\prod_{k=1}^{M} O_{a_{k}}\right\rangle-\prod_{k=1}^{M}\left\langle O_{a_{k}}\right\rangle\right|=\left|\sum_{n=1}^{M} r_{n} s_{n}\right| \leqslant \sum_{n=1}^{M}\left|r_{n}\right|\left|s_{n}\right| .
$$

Then, Eq. (11) follows from

$$
\left|s_{n}\right| \leqslant \prod_{k+1}^{M}\left\|O_{a_{k}}\right\|, \quad\left|r_{n}\right| \leqslant f_{A_{n}}\left(m_{n}\right) \prod_{k=1}^{n}\left\|O_{a_{k}}\right\|,
$$

where in the last inequality we have used (9), and the fact that $\|A B\| \leqslant\|A\|\|B\|$.

\section{B. Robustness against perturbations}

We are interested in seeing how the properties of the ground state subspace $\mathcal{S}_{X}$ change if we modify the states locally. In particular, we want to know the behavior of the expectation values of local observables:

$$
o_{a}(\epsilon):=\frac{\left\langle\Psi_{x}(\epsilon)\left|O_{a}\right| \Psi_{x}(\epsilon)\right\rangle}{\left\langle\Psi_{x}(\epsilon) \mid \Psi_{x}(\epsilon)\right\rangle}
$$

if we perturb every state $\left|\Psi_{x}\right\rangle \in \mathcal{S}_{X}$ as follows:

$$
\left|\Psi_{x}(\epsilon)\right\rangle=R_{X}(\epsilon)\left|\Psi_{x}\right\rangle
$$

in the limit $|X| \rightarrow \infty$, where $R_{X}(\epsilon)=R(\epsilon)^{\otimes|X|}$. Here, $R(\epsilon)=$ $\mathbb{1}+\epsilon Z$, where $Z$ is an operator acting on a single lattice site with $\|Z\|=1$, and $\epsilon$ sufficiently small. Note that we do not need to restrict ourselves to $Z$ acting on a single lattice site; in fact, we can always group spins into bigger spins and assume $Z$ acts on a single spin of the new lattice. Recall that these were exactly the natural perturbations in the context of PEPS. For simplicity in the notation we will restrict to the case of translational invariant perturbations, but the results hold true with the same proofs in the case of a site-dependent perturbation of the form $\otimes_{i \in X} R_{i}(\epsilon)|\Psi\rangle .{ }^{19}$

We will now show that LTQO implies robustness for local observables: If the observable $O_{a}$ satisfies LTQO, $o_{a}(\epsilon)$ is continuous at $\epsilon=0$, and its first derivative at that point is finite. Moreover, if not only the observable $O_{a}$ but also the single site region satisfies LTQO, then all higher order derivatives are also finite at $\epsilon=0$.

Proposition 6. If the observable $O_{a}$ satisfies LTQO, then $o_{a}(\epsilon)$ is continuous at $\epsilon=0$. More specifically, there exists a function $k_{A}(\epsilon) \rightarrow 0$ (as $\epsilon \rightarrow 0$ ) which is independent of $|X|$, such that

$$
\left|o_{a}(\epsilon)-o_{a}(0)\right| \leqslant\left\|O_{a}\right\| k_{A}(\epsilon)
$$

for all lattice sizes larger than some $\left|X_{\epsilon}\right|$.

Proof. The idea of the proof is to decompose the perturbation $R_{X}(\epsilon)$, Eq. (13), into two parts: One part is far away from $O_{a}$ and can thus be dealt with using LTQO, while the other part can be bounded directly as it only acts on a restricted region.

We start by choosing a number $m:=m(\epsilon)$ and a region $B_{m} \supset A$, such that the distance between $A$ and $\partial B_{m}$ is $m$. Define $\left|\Psi_{x}^{\prime}(\epsilon)\right\rangle=R_{X \backslash B_{m}}(\epsilon)\left|\Psi_{x}(0)\right\rangle$, i.e., $\left|\Psi_{x}(0)\right\rangle$ is only modified outside of region $B_{m}$. From now on we will omit the dependence of all the states and operators on $\epsilon$ to facilitate reading. We write the numerator of (12) as

$$
\left\langle\Psi_{x}\left|O_{a}\right| \Psi_{x}\right\rangle=\left\langle\Psi_{x}^{\prime}\left|O_{a}\right| \Psi_{x}^{\prime}\right\rangle+\left\langle\Psi_{x}^{\prime}\left|T_{a}\right| \Psi_{x}^{\prime}\right\rangle,
$$

where $T_{a}=R_{B_{m}}^{\dagger} O_{a} R_{B_{m}}-O_{a}$. In the same way, we replace the denominator by

$$
\left\langle\Psi_{x} \mid \Psi_{x}\right\rangle=\left\langle\Psi_{x}^{\prime} \mid \Psi_{x}^{\prime}\right\rangle+\left\langle\Psi_{x}^{\prime}\left|S_{a}\right| \Psi_{x}^{\prime}\right\rangle,
$$

where $S_{a}=R_{B_{m}}^{\dagger} R_{B_{m}}-\mathbb{1}$. With simple manipulations, we write

$$
\left|o_{a}(\epsilon)-o_{a}(0)\right| \leqslant\left|\frac{\left\langle\Psi_{x}^{\prime}\left|O_{a}\right| \Psi_{x}^{\prime}\right\rangle}{\left\langle\Psi_{x}^{\prime} \mid \Psi_{x}^{\prime}\right\rangle}-o_{a}(0)\right|+h(\epsilon),
$$

where

$$
h(\epsilon)=\frac{\left\|O_{a}\right\|\left\|S_{a}\right\|+\left\|T_{a}\right\|}{1-\left\|S_{a}\right\|} .
$$

In order to bound this term, we write $T_{a}=\left(R_{B_{m}}-\right.$ $\mathbb{1})^{\dagger} O_{a}\left(R_{B_{m}}-\mathbb{1}\right)+\left(R_{B_{m}}-\mathbb{1}\right)^{\dagger} O_{a}+O_{a}\left(R_{B_{m}}-\mathbb{1}\right)$, so that

$$
\left\|T_{a}\right\| \leqslant\left\|O_{a}\right\|\left\|R_{B_{m}}-\mathbb{1}\right\|\left(2+\left\|R_{B_{m}}-\mathbb{1}\right\|\right) .
$$

The same bound applies to $\left\|S_{a}\right\|$ when replacing $\left\|O_{a}\right\|$ by 1 . Using the binomial expansion of $R_{B_{m}}$, we have

$$
\left\|R_{B_{m}}-\mathbb{1}\right\| \leqslant(1+|\epsilon|)^{(|A|+2 m)^{2}}-1,
$$

where we have used that $\left|B_{m}\right| \leqslant(|A|+2 m)^{2}$. Choosing $m(\epsilon)=|\epsilon|^{-1 / 2+x}$ with $x \in(0,1 / 2)$, we have $\left\|R_{B_{m}}-\mathbb{1}\right\| \rightarrow 0$ in the limit $\epsilon \rightarrow 0$, and thus $h(\epsilon) \rightarrow 0$. Finally, using (8) we can bound the first term of (16) by $\left\|O_{a}\right\| f_{A}(m)$, which vanishes in that limit as well.

Proposition 7. If an observable $O_{a}$ satisfies LTQO, then $d o_{a}(\epsilon) / d \epsilon$ is finite at $\epsilon=0$. Formally, the limit

$$
\left.\lim _{|X| \rightarrow \infty} \frac{d o_{a}(\epsilon)}{d \epsilon}\right|_{\epsilon=0}
$$

exists and is finite.

Proof. In order to determine $o_{a}^{\prime}=d o_{a}(\epsilon) / d \epsilon$ at $\epsilon=0$, we first have to take the derivative of $|\Psi(\epsilon)\rangle$ as given in (13). We split $o_{a}^{\prime}$ into two parts: (i) one part corresponding to the derivative involving lattice sites included in $A$; (ii) the rest. The first is obviously finite. The second can be written as $\tilde{o}_{a}^{\prime}$, where

$$
\tilde{o}_{a}(\epsilon)=\frac{\left\langle\Psi_{x}\left|\tilde{R}(\epsilon) O_{a}\right| \Psi_{x}\right\rangle}{\left\langle\Psi_{x}|\tilde{R}(\epsilon)| \Psi_{x}\right\rangle}
$$

with $\tilde{R}(\epsilon)=(\mathbb{1}+\epsilon W)^{\otimes|X \backslash A|}$ and $W=Z+Z^{\dagger}+\epsilon Z^{\dagger} Z$. Taking the derivative and setting $\epsilon=0$ we obtain

$$
\tilde{o}_{a}^{\prime}=\sum_{n \notin A}\left[\left\langle W_{n} O_{a}\right\rangle-\left\langle W_{n}\right\rangle\left\langle O_{a}\right\rangle\right],
$$

where the sum is extended to all sites not belonging to $A, W_{n}$ denotes $W$ acting on site $n$, and the expectation values are taken in the (normalized) state $\left|\Psi_{x}\right\rangle$. Using that the correlation functions decay faster than any polynomial, Eq. (9), the sum converges in the limit $|X| \rightarrow \infty$.

One can extend the proof to any higher order derivative.

Proposition 8 . If both an observable $O_{a}$ and the single site region satisfy LTQO, then

$$
\left.\lim _{|X| \rightarrow \infty} \frac{d^{n} o_{a}(\epsilon)}{d \epsilon^{n}}\right|_{\epsilon=0}
$$

exists and is finite. 
The proof is analogous to the one above, although a bit more involved. It relies again on the fact that connected correlation functions decay sufficiently fast, Eq. (11).

Note that to prove the finiteness of the derivatives, we only use the decay of the correlation functions. The full power of the LTQO condition is only used directly in the continuity proof. This is a formal proof in this context that with exponential decay of correlations, one can only expect first order or infiniteorder phase transitions. Having LTQO rules out the first-order ones.

If we do not have LTQO for the single site region and we have it only for a particular observable $O_{a}$, we cannot guarantee Proposition 8 to hold. In this case, we can only deduce continuity and bounded first derivative. This rules out first and second order phase transitions witnessed by $O_{a}$, but leaves open the possibility of higher-order ones. Finally, note that only the weakest condition (8) has been used for the proofs in this section.

\section{LTQO IN PEPS}

We have seen that LTQO ensures stability for PEPS under a class of natural perturbations. In this section, we will analyze how to detect LTQO in PEPS, and discuss PEPS-specific implications of the stability condition.

\section{A. Detecting LTQO in PEPS}

Consider a translationally invariant PEPS $\left|\Psi_{x}\right\rangle$ (see upperleft part of Fig. 4) with some boundary condition (which by the very definition of LTQO will play no role). The PEPS is fully characterized by a tensor $A$ with some physical index $n=1, \ldots, d$ and auxiliary indices $\alpha_{k}=1, \ldots, D$, where $d$ is the dimension of the spin and $D$ the bond dimension. In order to investigate the LTQO property for this state, we consider an observable $O_{1}$, with $\left\|O_{1}\right\|=1$, acting on the central spin in

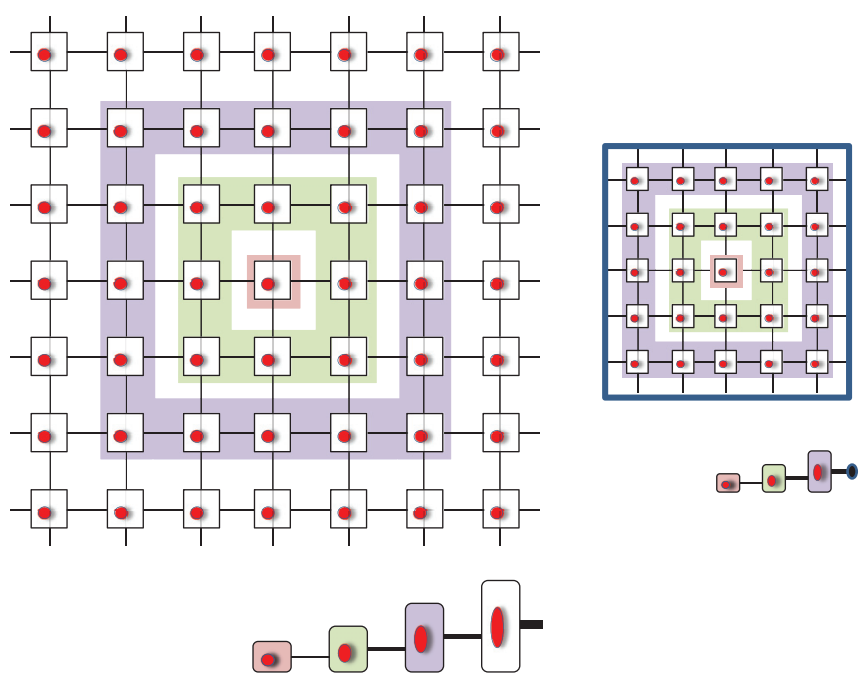

FIG. 4. (Color online) Verifying LTQO in PEPS. Left: By consecutively blocking regions around the central spin, we can map the PEPS onto a one-dimensional matrix product state (MPS). Right: The effect of the boundary condition (dark blue) on the central spin can be mapped to an eigenvalue problem for the transfer operators of the one-dimensional chain (see text). the figure, which we will call spin 1; note that we can always block spins such that the operator $O_{1}$ only acts on a single effective spin. We now define a one-dimensional structure of tensors by layer-wise blocking tensors around spin 1: The first tensor corresponds to spin 1 itself. The second is obtained by contracting all tensors around spin 1 (marked green in Fig. 4). The third one contains those next to the previous layer (marked violet in Fig. 4), and so on. The resulting chain of tensors is represented in the lower-left part of Fig. 4 . We denote them by $B[1], B[2], \ldots, B[m]$, where the dimensions of the physical and the auxiliary indices now grow with the layer $m$. That is, in this representation the PEPS $\left|\Psi_{x}\right\rangle$ has the form of a (non-translationally-invariant) matrix product state (MPS).

Let us now consider a region $B$ in the original lattice centered around spin 1, containing layers 1 to $m$. Any state $\left|\Psi_{b}\right\rangle \in \mathcal{S}_{B}$ can be obtained by contracting those layers with an arbitrary tensor on its boundary (see upper-right part of Fig. 4). In terms of the MPS representation (lower part), this just corresponds to contracting a vector with the auxiliary index on the right. Thus,

$$
\left|\Psi_{R}\right\rangle_{B}=\sum_{n_{1}, \ldots, n_{m}}\left(B[1]^{n_{1}}\left|\cdots B[m]^{n_{m}}\right| R\right)\left|n_{1}, \ldots, n_{m}\right\rangle,
$$

where $B[k]^{n}$ are $D_{k-1} \times D_{k}$ matrices, and $\left.\mid B[1]^{n}\right)$ and $\left.\mid R\right)$ are vectors of dimensions $D_{1}$ and $D_{m}$, respectively (we have used curly brackets to denote vectors acting on the auxiliary indices in order to avoid confusion with the physical spin degrees of freedom). As is standard in MPS theory, in order to determine expectation values of local observables acting on spin 1 , it is convenient to define the following completely positive maps:

$$
\begin{aligned}
& \left.\mathcal{E}_{1}(X)=\sum_{i_{1}, j_{1}} \mid B^{j_{1}}\right)\left\langle j_{1}|X| i_{1}\right\rangle\left(B^{i_{1}} \mid,\right. \\
& \mathcal{E}_{n}(X)=\sum_{i_{n}} B^{i_{n} \dagger} X B^{i_{n}}
\end{aligned}
$$

for $n=2, \ldots, m$, as well as,

$$
\mathcal{M}_{m}=\mathcal{E}_{m} \circ \ldots \mathcal{E}_{2} \circ \mathcal{E}_{1} .
$$

We can thus write:

$$
\frac{\left\langle\Psi_{R}\left|O_{1}\right| \Psi_{R}\right\rangle}{\left\langle\Psi_{R} \mid \Psi_{R}\right\rangle}=\frac{\left(R\left|\mathcal{M}_{m}\left(O_{1}\right)\right| R\right)}{\left(R\left|\mathcal{M}_{m}(\mathbb{1})\right| R\right)} .
$$

We will have LTQO for $O_{1}$ whenever this quantity becomes independent of the vector $\mid R$ ) in the limit $m \rightarrow \infty$ via a rapidly decaying function $f(m)$.

In order to numerically verify the presence of LTQO using Eq. (23), one finds the maximum and minimum generalized eigenvalues $\lambda\left(O_{1}\right)$ of the eigenvalue equation

$$
\left.\left.\mathcal{M}_{m}\left(O_{1}\right) \mid R\right)=\lambda\left(O_{1}\right) \mathcal{M}_{m}(\mathbb{1}) \mid R\right),
$$

which can be done using Lanczos methods, together with approximate contraction of the quasi-1D tensor network. Defining

$$
\epsilon_{m}=\lambda_{\max }\left(O_{1}\right)-\lambda_{\min }\left(O_{1}\right),
$$

we then have

$$
\left|\frac{\left\langle\Psi_{R}\left|O_{1}\right| \Psi_{R}\right\rangle}{\left\langle\Psi_{R} \mid \Psi_{R}\right\rangle}-\frac{\left\langle\Psi_{S}\left|O_{1}\right| \Psi_{S}\right\rangle}{\left\langle\Psi_{S} \mid \Psi_{S}\right\rangle}\right| \leqslant \epsilon_{m}
$$


and thus, it only remains to check that $\epsilon_{m}$ decays sufficiently fast with $m .^{20}$

\section{B. Implications of LTQO for PEPS simulations}

If a PEPS possesses LTQO for a certain local observable $O_{1}$, this implies that in order to compute expectation values of $O_{1}$, we can choose any boundary condition $\mid R$ ) we like; in particular, we can choose $\mid R$ ) to be a product state. If the boundary is at a distance $m$ from the observable, this implies that the boundary as seen by the observable is a matrix product operator (MPO) with bond dimension $D^{2 m}$ (obtained by blocking the tensors in Fig. 4 in radial slices). In particular, if $f(m)=O\left(e^{-\alpha m}\right)$, then the bond dimension required to compute the value of $\left\langle O_{1}\right\rangle$ in the thermodynamic limit up to precision $\epsilon$ scales polynomially in $1 / \epsilon$. Thus, LTQO provides a formal justification of the approximate PEPS contraction scheme in which the boundary is approximated by an MPO at every step. ${ }^{21}$

\section{STABILITY OF LTQO}

In this section, we prove that in the presence of a spectral gap, the LTQO condition for a system can only disappear when closing the (local) gap. This is important since it allows us to infer LTQO for a whole neighborhood of systems rather than only for isolated points in Hamiltonian space.

Given a system $H_{X}=\sum_{x \in X} h_{x}$, we say that it has local gap if there exists a constant $\gamma>0$ such that for all $|X|$ and all spherical regions $R \subset X$, the Hamiltonian $H_{R}=\sum_{x \in R} h_{x}$ has a spectral gap at least $\gamma$ above the ground state energy.

Theorem 9. Consider a Hamiltonian $H_{X}=\sum_{x} h_{x}$ which is short ranged and frustration free ${ }^{22}$ (but not necessary translationally invariant), and let $\epsilon_{x} \geqslant 0$ for all $x \in X$. Assume that (i) the system $H_{X}$ has LTQO with some superpolynomially decaying function $\hat{f}(m)$; (ii) for all $0 \leqslant \delta_{x} \leqslant \epsilon_{x}$, the perturbed Hamiltonian

$$
H_{X}^{\vec{\delta}}=\sum_{x} h_{x}+\delta_{x} k_{x}
$$

has uniform (in $\delta$ ) local gap and $k_{x}$ acts on the same sites as $h_{x}$, where we assume $\left\|h_{x}\right\|,\left\|k_{x}\right\| \leqslant 1$ for all $x$. Then, the perturbed system $H_{X}^{\vec{\epsilon}}$ has LTQO.

It is crucial for the proof that we assume LTQO for the whole system, and not just for a particular region or observable. To prove the result, we will use the following result from Ref. 23.

Lemma 10. (Theorem 3.4 in Ref. 23) Let $Y$ be any region of a system $X$ and $Y_{R}$ the region enlarged by sites at distance $\leqslant R$ from $Y$. Consider a smooth path of Hamiltonians on $X, H(s)=$ $H_{0}+\Phi(s), 0 \leqslant s \leqslant 1$, with uniformly bounded local terms, bounded derivatives, and a uniform lower bound on the spectral gap, and for which $\Phi(s)$ is supported on $Y$. Let $P_{0}$ and $P_{1}$ be the projector onto the ground space of $H(0)$ and $H(1)$, respectively. Then, there exists a unitary operation $V_{R}$ acting on $Y_{R}$ such that (in operator norm)

$$
\left\|P_{0}-V_{R} P_{1} V_{R}^{\dagger}\right\| \leqslant \tilde{f}(R),
$$

where $\tilde{f}(m)$ decays superpolynomially.

Note that it will be decisive that the Lemma makes no assumption about the rank of $P_{0}$ and $P_{1}$.
Proof of Theorem 9. In order to prove LTQO for the deformed system (24), we consider a spherical region $A$ and subsequently add concentric rings $B, C, D$, such that their boundaries are separated by $\frac{m}{3}$. We denote the union of the regions $A, B, C, D$ by $Y$, and the projector onto the ground space of the original Hamiltonian $H_{X}$ in region $Y$ by $P$.

Let $P^{A B}$ denote the projection onto the ground space of Hamiltonian

$$
H_{A B}^{\vec{\delta}}=\sum_{x \in X} h_{x}+\sum_{x \in A, B} \delta_{x} k_{x},
$$

i.e., where the perturbation only acts in regions $A$ and $B$. Since we assume a local gap in the theorem, Lemma 10 implies the existence of a unitary $V_{A B C}$ (supported on regions $A, B, C$ ) such that

$$
\left\|P^{A B}-V_{A B C} P V_{A B C}^{\dagger}\right\| \leqslant \tilde{f}\left(\frac{m}{3}\right) .
$$

Using successive triangle inequalities, the submultiplicativity and unitary invariance of the operator norm, and Eq. (25), we find (with $c=\frac{\operatorname{tr} P V_{A B C}^{\dagger} O_{A} V_{A B C}}{\operatorname{tr} P}$ )

$$
\begin{aligned}
&\left\|P^{A B} O_{A} P^{A B}-c P^{A B}\right\| \\
& \leqslant\left\|V_{A B C} P V_{A B C}^{\dagger} O_{A} V_{A B C} P V_{A B C}^{\dagger}-c V_{A B C} P V_{A B C}^{\dagger}\right\| \\
&+3\left\|O_{A}\right\| \tilde{f}\left(\frac{m}{3}\right) .
\end{aligned}
$$

The left part can be further bounded using the LTQO condition for $H_{X}$ which says that $\left\|P O_{A B C} P-c^{\prime} P\right\| \leqslant \hat{f}\left(\frac{m}{3}\right)\left\|O_{A B C}\right\|$ for all $O_{A B C}$ supported in the union of regions $A, B, C$ (in particular for $\left.O_{A B C}=V_{A B C}^{\dagger} O_{A} V_{A B C}\right)$, where $c^{\prime}=\frac{\operatorname{tr} P O_{A B C}}{\operatorname{tr} P} \equiv$ $c$, which yields

$$
\begin{aligned}
& \left\|P^{A B} O_{A} P^{A B}-c P^{A B}\right\| \\
& \quad \leqslant\left\|O_{A}\right\| \hat{f}\left(\frac{m}{3}\right)+3\left\|O_{A}\right\| \tilde{f}\left(\frac{m}{3}\right) \leqslant 4\left\|O_{A}\right\| f\left(\frac{m}{3}\right),
\end{aligned}
$$

where $f$ is a superpolynomially decaying upper bound to $\tilde{f}$ and $\hat{f}$.

Another application of Lemma 10 proves the existence of a unitary $V_{B C D}$ such that

$$
\left\|P^{A B C D}-V_{B C D} P^{A B} V_{B C D}^{\dagger}\right\| \leqslant f\left(\frac{m}{3}\right),
$$

where $P^{A B C D}$ is the projector onto the ground space of $H_{X}^{\vec{\delta}}$. Again,

$$
\begin{aligned}
\left\|P^{A B C D} O_{A} P^{A B C D}-c P^{A B C D}\right\| & \\
\leqslant & \| V_{B C D} P^{A B} V_{B C D}^{\dagger} O_{A} V_{B C D} P^{A B} V_{B C D}^{\dagger} \\
& -c V_{B C D} P^{A B} V_{B C D}^{\dagger}\|+3\| O_{A} \| f\left(\frac{m}{3}\right) .
\end{aligned}
$$

Since in the first term on the r.h.s., $O_{A}$ commutes with $V_{B C D}$, $V_{B C D}^{\dagger} V_{B C D}$ cancels, and we find (using unitary invariance of 
the norm)

$$
\begin{aligned}
& \left\|P^{A B C D} O_{A} P^{A B C D}-c P^{A B C D}\right\| \\
& \quad \leqslant\left\|P^{A B} O_{A} P^{A B}-c P^{A B}\right\|+3\left\|O_{A}\right\| f\left(\frac{m}{3}\right) \\
& \quad \leqslant 7\left\|O_{A}\right\| f\left(\frac{m}{3}\right),
\end{aligned}
$$

with $c$ defined as above. Using the characterization given in Corollary 3 of Ref. 1, one can see that the actual value of the constant $c$ plays no role in the definition of LTQO, and therefore, we have shown LTQO for the perturbed system.

This result can be used to construct new examples of systems verifying LTQO. For instance, it is shown in Appendix E of Ref. 8 that if we start with a system with LTQO and made out of commuting terms (such as the toric code or quantum double models), small perturbations of the type (3) verify the hypothesis of the theorem. In this way, we can give the first 2D examples of systems with noncommuting Hamiltonians satisfying LTQO.

\section{CONCLUSIONS}

In this paper, we have analyzed the stability of a PEPS under physical perturbations to the local tensor which defines it. We have shown how restricting the LTQO condition $^{1}$ to particular observables and regions gives a checkable criterion which makes this assignment between the PEPS and the local tensor robust. This robustness translates then to any situation in which this assignment is exploited, with examples ranging from classifying quantum phases in locally interacting spin systems $^{4,8}$ to approximating numerically ground states of $2 \mathrm{D}$ local Hamiltonians. ${ }^{24}$

\section{ACKNOWLEDGMENTS}

We are indebted to Frank Verstraete for many useful comments on this work. J.I.C. acknowledges support by the EU project AQUTE, the DFG SFB 631 and Exzellenzcluster NIM, and Catalunya Caixa. S.M. acknowledges funding provided by the Institute for Quantum Information and Matter, an NSF Physics Frontiers Center with support of the Gordon and Betty Moore Foundation through Grant No. GBMF1250 and by the AFOSR Grant No. FA8750-12-2-0308. D.P.G. acknowledges support from Spanish Grant Nos. MTM201126912 and QUITEMAD, and European CHIST-ERA project CQC. N.S. acknowledges support by the Alexander von Humboldt foundation.

\section{APPENDIX A: LTQO FOR INJECTIVE MPS}

In this appendix, we give a formal proof of the following theorem:

Theorem 11. Parent Hamiltonians of translationally invariant, injective MPS satisfy LTQO.

Among MPS experts the above result has been known for some time, but we think that a rigorous proof would illuminate some of the key aspects of LTQO as it relates to the concept of insensitivity of the bulk to boundary conditions. Before proving the result, we recall the requisite basic machinery from the
MPS literature. In particular, we note that a translationally invariant MPS is equivalent to a $1 \mathrm{D}$ PEPS. It is, hence, given by a collection of $D \times D$ matrices $\left(A_{i}\right)_{i=1}^{d}$, with $d$ the local physical dimension. Since contraction in this case reduces to matrix multiplication, for each chain with $N$ spins, the MPS reads:

$$
|\psi\rangle=\sum_{i_{1}, \ldots, i_{N}=1}^{d} \operatorname{tr}\left(A_{i_{1}} \cdots A_{i_{N}}\right)\left|i_{1} \cdots i_{N}\right\rangle .
$$

An MPS is called injective if there exists a length $R \geqslant 1$, such that the map

$$
K_{R}(X): X \mapsto \sum_{i_{1}, \ldots, i_{R}=1}^{d} \operatorname{tr}\left(X A_{i_{1}} \cdots A_{i_{R}}\right)\left|i_{1} \cdots i_{R}\right\rangle
$$

is injective. The minimal such $R$ is called the injectivity length. By the quantum Wielandt inequality of Ref. 25, the injectivity length is known to be upper-bounded by $\left(D^{2}-d+1\right) D^{2}$. Hence by blocking at most $\left(D^{2}-d+1\right) D^{2}$ spins we can assume without loss of generality that $R=1$. Injective MPS are the unique ground states of their parent Hamiltonians, ${ }^{16,26}$ which have a uniform gap above the ground state. ${ }^{26}$ Moreover, parent Hamiltonians of injective MPS also verify the local-gap condition of Ref. 1 . That is, for any region of $L$ consecutive spins, the Hamiltonian $H_{L}=\sum_{i=1}^{L-1} h_{i, i+1}$, whose ground-state subspace is

$$
\operatorname{ker}\left(H_{L}\right)=\left\{K_{L}(X) \mid X \in M_{D \times D}\right\},
$$

has a uniform (in $L$ ) spectral gap. ${ }^{26}$ This allows us to conclude from Theorem 11 and the main result in Ref. 1 that:

Corollary 12. Parent Hamiltonians of translationally invariant, injective MPS have a stable spectral gap against arbitrary quasilocal perturbations.

Note that the above corollary combined with the quasiadiabatic continuation technique ${ }^{23,27}$ implies stability of the ground-state subspace with respect to properties of local observables.

To show Theorem 11, we will rely on the canonical form of MPS stated in Ref. 16. Any injective MPS can be represented by a set of $D \times D$ matrices $\left\{A_{i}\right\}_{i=1}^{d}$, such that the completely-positive and trace-preserving map $\mathcal{E}$ given by $\mathcal{E}(X)=\sum_{i=1}^{d} A_{i} X A_{i}^{\dagger}$, has a nondegenerate eigenvalue of modulus 1 corresponding to $\Lambda$, where $\Lambda$ is a diagonal, positive, full-rank matrix with $\operatorname{tr}(\Lambda)=1$. If we denote the second largest (in magnitude) eigenvalue of $\mathcal{E}$ as $\lambda_{2}$, then it follows that the map $\mathcal{E}$ has a spectral gap given by $1-\left|\lambda_{2}\right|$.

Proof of Theorem 11. We consider a region $B$ with spins $1, \ldots, 2 m+l$ and region $A$ with spins $m+1, \ldots, m+l$ as well as an unnormalized ground state of $H_{B}$ given by $X$ :

$$
\left|\psi_{X}\right\rangle=\sum_{i_{1}, \ldots, i_{2 m+l}=1}^{d} \operatorname{tr}\left(X A_{i_{1}} \cdots A_{i_{2 m+l}}\right)\left|i_{1} \cdots i_{2 m+l}\right\rangle .
$$

To show LTQO it is enough to prove that for any observable $O_{A}$ acting on region $A$ :

$$
\left|\frac{\left\langle\psi_{X}\left|O_{A}\right| \psi_{X}\right\rangle}{\left\langle\psi_{X} \mid \psi_{X}\right\rangle}-\operatorname{tr}\left(\mathcal{E}_{O_{A}}(\Lambda)\right)\right| \leqslant\left\|O_{A}\right\| f(m)
$$


with $f(m)$ exponentially decaying in $m$ and

$$
\mathcal{E}_{O_{A}}(X)=\sum_{i_{m+1}, \ldots, i_{m+l}, j_{m+1}, \ldots, j_{m+l}=1}^{d}\left\langle j_{m+1} \cdots j_{m+l}\left|O_{A}\right| i_{m+1} \cdots i_{m+l}\right\rangle A_{i_{m+1}} \cdots A_{i_{m+l}} X A_{j_{m+l}}^{\dagger} \cdots A_{j_{m+1}}^{\dagger} .
$$

Set $g\left(O_{A}\right)=\left|\frac{\left\langle\psi_{X}\left|O_{A}\right| \psi_{X}\right\rangle}{\operatorname{tr}\left(X X^{\dagger} \Lambda\right)}-\operatorname{tr}\left(\mathcal{E}_{O_{A}}(\Lambda)\right)\right|$. It is shown in Lemma 5.2.(2) of Ref. 26 that $g\left(O_{A}\right) \leqslant\left\|O_{A}\right\| f(m)$ with $f$ exponentially decaying with $m$. Then,

$$
\begin{aligned}
\left|\frac{\left\langle\psi_{X}\left|O_{A}\right| \psi_{X}\right\rangle}{\left\langle\psi_{X} \mid \psi_{X}\right\rangle}-\operatorname{tr}\left(\mathcal{E}_{O_{A}}(\Lambda)\right)\right| & \leqslant\left|\frac{\left\langle\psi_{X}\left|O_{A}\right| \psi_{X}\right\rangle}{\left\langle\psi_{X} \mid \psi_{X}\right\rangle}-\frac{\left\langle\psi_{X}\left|O_{A}\right| \psi_{X}\right\rangle}{\operatorname{tr}\left(X X^{\dagger} \Lambda\right)}\right|+\left|\frac{\left\langle\psi_{X}\left|O_{A}\right| \psi_{X}\right\rangle}{\operatorname{tr}\left(X X^{\dagger} \Lambda\right)}-\operatorname{tr}\left(\mathcal{E}_{O_{A}}(\Lambda)\right)\right| \\
& \leqslant\left\|O_{A}\right\| g(\mathbb{1})+g\left(O_{A}\right) \leqslant 2\left\|O_{A}\right\| f(m),
\end{aligned}
$$

as desired.

${ }^{1}$ S. Michalakis and J. Pytel, Commun. Math. Phys. 322, 277 (2013). ${ }^{2}$ S. Bravyi, M. Hastings, and S. Michalakis, J. Math. Phys. 51, 093512 (2010).

${ }^{3}$ S. Bravyi and M. B. Hastings, Commun. Math. Phys. 307, 609 (2011).

${ }^{4}$ N. Schuch, I. Cirac, and D. Pérez-García, Ann. Phys. 325, 2153 (2010).

${ }^{5}$ D. Perez-Garcia, M. Sanz, C. E. Gonzalez-Guillen, M. M. Wolf, and J. I. Cirac, New J. Phys. 12, 025010 (2010).

${ }^{6}$ F. Pollmann, A. M. Turner, E. Berg, and M. Oshikawa, Phys. Rev. B 81, 064439 (2010).

${ }^{7}$ X. Chen, Z.-C. Gu, and X.-G. Wen, Phys. Rev. B 83, 035107 (2011).

${ }^{8}$ N. Schuch, D. Perez-Garcia, and I. Cirac, Phys. Rev. B 84, 165139 (2011).

${ }^{9}$ X. Chen, Z.-X. Liu, and X.-G. Wen, Phys. Rev. B 84, 235141 (2011).

${ }^{10}$ The intersection property can be deduced from the injectivity, or more generally, $G$-injectivity ${ }^{4,13}$ property of a PEPS, but it is less restrictive, as shown for instance in Ref. 13.

${ }^{11}$ This way, we keep the states $\left|\psi_{b}\right\rangle$, considered below in the definition of LTQO, under control.

${ }^{12} \mathrm{We}$ conjecture, based on the intuition built in Refs. 14 and 15 , that the natural perturbations defined in Definition 1 are exactly those of the type (1) with the added constraint that they lead to a continuous change in the parent Hamiltonian. Beyond particular classes of PEPS, like injective ones (where the result is trivial) or MPS [where one may use the canonical form (Ref. 16) to prove it], we do not have at this point a complete result connecting (1) with (2).

${ }^{13}$ D. Perez-Garcia, F. Verstraete, J. I. Cirac, and M. M. Wolf, Quantum Inf. Comput. 8, 0650 (2008).

${ }^{14}$ C. Fernández-González, N. Schuch, M. M. Wolf, J. I. Cirac, and D. Pérez-García, Phys. Rev. Lett. 109, 260401 (2012).

${ }^{15}$ C. Fernandez-Gonzalez, N. Schuch, M. M. Wolf, J. I. Cirac, and D. Perez-Garcia, arXiv:1210.6613.
${ }^{16}$ D. Perez-Garcia, F. Verstraete, M. M. Wolf, and J. I. Cirac, Quant. Inf. Comput. 7, 401 (2007).

${ }^{17}$ N. Schuch, D. Poilblanc, J. I. Cirac, and D. Pérez-García, Phys. Rev. B 86, 115108 (2012).

${ }^{18}$ The speed of the decay in the correlations is the same that appears in the definition of LTQO.

${ }^{19}$ There is another motivation for this type of perturbation that goes beyond PEPS. Imagine each spin is weakly coupled to a local environment. After the action of the noise for time $\epsilon$, the system is in a convex combination of states of the form $\otimes_{i \in X} R_{i}(\epsilon)|\Psi\rangle$, where each $R_{i}(\epsilon)$ is $\epsilon$-close to the identity. Hence, LTQO implies that the system is also stable against this type of dissipative noise.

${ }^{20}$ Note that $\epsilon_{m}$ depends on the chosen observable. If we want to check LTQO for the one-site region, we have to maximize $\epsilon_{m}$ among all possible observables $O_{1}$ with $\left\|O_{1}\right\|=1$.

${ }^{21} \mathrm{~F}$. Verstraete and J. I. Cirac, arXiv:cond-mat/0407066.

${ }^{22}$ Though we assume it for simplicity, the hypothesis of frustration freeness can be relaxed. The notion of LTQO for frustrated Hamiltonians with a low-energy subspace of energy-splitting $\epsilon$ separated by a uniform gap $\gamma \gg \epsilon$ from the rest of the spectrum, is well defined if one assumes the existence of subregions with low-energy subspaces separated by local gaps to the rest of the spectrum. In that case, LTQO is defined with respect to the projections onto the local, low-energy subspaces with energy below $\epsilon$, instead of the exact ground-state subspace of frustration-free Hamiltonians.

${ }^{23}$ S. Bachmann, S. Michalakis, B. Nachtergaele, and R. Sims, Commun. Math. Phys. 309, 835 (2012).

${ }^{24}$ J. Jordan, R. Orus, G. Vidal, F. Verstraete, and J. I. Cirac, Phys. Rev. Lett. 101, 250602 (2008).

${ }^{25}$ M. Sanz, D. Perez-Garcia, M. M. Wolf, and J. I. Cirac, IEEE Trans. Inf. Theory 56, 4668 (2009).

${ }^{26}$ M. Fannes, B. Nachtergaele, and R. F. Werner, Commun. Math. Phys. 144, 443 (1992).

${ }^{27}$ M. B. Hastings and X.-G. Wen, Phys. Rev. B 72, 045141 (2005). 